\title{
13 Qualität ist, was der virtuelle Kunde will - Fehlende Kundenorientierung als hausgemachtes Problem
}

\author{
Thomas Breisach
}

\section{Bestandsaufnahme und Stellenwert}

Der Weg ist das Ziel. Eine Weisheit, die leider auch für viele Verantwortlichen in der Gesundheitskommunikation gilt. Der Grund ist ganz einfach: Nur bei wenigen Herstellern, Leistungserbringern und -erstattern steht das echte Informationsverhalten und das genaue Interesse der Kunden oder der Zielgruppe im Vordergrund, was zumeist an der dazu fehlenden Information liegt. Häufig wird daher aus der Perspektive und Erwartungshaltung des Senders kommuniziert und man gibt sich bereits damit zufrieden, wenn man es geschafft hat, verschiedene und noch dazu neue Instrumente eingesetzt zu haben. Die Frage, ob dies zielführend ist oder nicht, gerät dabei häufig in den Hintergrund. Dies ist jedoch kein Spezifikum für die digitale Kommunikation, sondern lässt sich auf den gesamten Bereich der Gesundheitskommunikation ausdehnen (Breisach 2016). Häufig sind nichtbetroffene Meinungsführer der Überzeugung, die Bedürfnisse der Betroffenen zu kennen und im Sinn der potenziellen Zielgruppe zu kommunizieren, ohne dabei jedoch deren eigentliches Informationsverhalten und Sprache zu berücksichtigen. Dass es sich hierbei um ein klassisches Problem handelt, zeigen bereits Beispiele aus der Antike. So haben bereits vor mehr als 2000 Jahren im alten Griechenland und Rom gut situierte und gebildete Männer, wie Pseudo-Plutarch oder Tacitus, die richtige Ernährung für schwangere Frauen und stillende Mütter diskutiert, ohne die betroffene Zielgruppe in Ihre Wissensbildung und in die Kommunikation miteinzubeziehen. Man vernachlässigte auch die Tatsache, dass es auch damals die sozialen und regionalen Unterschiede waren, die das Wissen und Verhalten der Mütter und somit auch die Ernährung der Neugeborenen beeinflussten (Wiesehöfer 2008). Wenn es also darum geht, dieses 
nichtpatientenorientierte Verhalten zu vermeiden, dann muss in der Sprache kommuniziert werden, die die Zielgruppe versteht, und zudem auf eine dialogorientierte Kommunikation gesetzt werden, die über die Kanäle stattfindet, in denen sich die Zielgruppe informiert und das ist heute zweifelsfrei der Gesamtbereich der digitalen Kommunikation.

\section{Beschreibung des Transformationsprozesses}

Die Bedeutung des Internets als relevante Informationsquelle zu Fragen der Gesundheit wächst immens. Es hat sich in den letzten Jahren für Verbraucher zu einer der wichtigsten Quellen für Gesundheitsinformationen entwickelt und sich bei den Informationssuchenden in Deutschland etabliert. Bereits 2010 nutzten laut einer von der FORSA für die BITKOM erstellten Studie 19 Millionen Deutsche das Internet, um Gesundheitsinformationen zu finden. Dabei sind die Hauptgründe für die stetig weiterwachsende Nutzung des Internet Aktualität, Detailliertheit und persönliche Relevanz, die diesem Medium in Bezug auf Gesundheitsfragen eher zugesprochen werden als anderen Quellen (Schachinger 2014).

Während es früher neben Ärzten hauptsächlich das direkte persönliche Umfeld war, das bei Fragen rund um Erkrankungen und Medikation zu Rate gezogen wurde, so sind es heute verstärkt die sozialen Medien, in denen nach Rat, sowie persönlichen Erfahrungen anderer direkt oder indirekt Betroffener gesucht wird. Bereits 2011 konnten im Rahmen einer Gemeinschaftsstudie von azionare und der Vendus-Gruppe dabei deutliche Unterschiede im Nutzungsverhalten der digitalen Patienten abhängig von der jeweiligen Indikation nachgewiesen werden. Der größte Diskussionsbedarf in sozialen Medien, worunter nicht nur soziale Netzwerke wie Facebook verstanden wurden, sondern auch sämtliche Webseiten, die ein Forum bzw. eine Dialogmöglichkeit anboten, bestand demnach bei den Indikationen Allergie, Depression und Aufmerksamkeitsdefizitsyndrom (ePatient Monitor 2011, 169). Das Ergebnis gewinnt aber erst dadurch deutlich an Relevanz, indem man auch die Social-MediaAffinität der jeweiligen Betroffenen und die Reichweite der Beiträge berücksichtigt. Während auch hier Aufmerksamkeitsdefizitsyndrom den Spitzenplatz belegt, kam es zu einer Veränderung auf den Plätzen, welche nun von den an den Themen Brustkrebs und Darmkrebs Interessierten übernommen wurden. Aber auch die Betroffenen und Angehörigen der Indikationen Multiple Sklerose oder Parkinson wiesen eine deutlich höhere Social-Media-Affinität aus. Falls der Dialog mit den Betroffenen im Vordergrund stehen soll, dann eigneten sich 2011 am besten allergisches Asthma, Depression und Diabetes (ePatient Monitor 2011, 17of.). Andere Indikationen zeichneten sich dagegen durch eine sehr geringe Diskussionsbereitschaft aus, was zumindest Social-Media-Ansätze als wenig vielversprechend erscheinen lässt. Zudem zeichnete sich in den letzten Jahren eine neue Gruppe von Patienten ab, die sich im Internet sehr intensiv mit Ihrer Erkrankung auseinandersetzen und als Patient Opinion Leader (POL) eine hohe Glaubwürdigkeit bei anderen Betroffenen besitzen.

\section{Chancen und Risiken}

Mit der Etablierung des Internets als Informationsquelle zu Gesundheitsfragen und der Dominanz der Smartphones ist zugleich ein gewaltiger Markt für Gesundheits- 
Apps entstanden. Dies erscheint nachvollziehbar, wenn man die wachsende Dominanz der Smartphones in Deutschland betrachtet. Aktuelle Zahlen zeigen, dass die Nutzung von Desktop-PCs leicht rückläufig ist, während der Anteil der Nutzer von Smartphones weiter deutlich steigt und diese bis 2021 weltweit zum wichtigsten Onlinezugang werden. (Brandt 2017). So nutzten im zweiten Halbjahr 2016 bereits 55 Prozent der Bevölkerung der Bundesrepublik Deutschland Smartphones, wohingegen lediglich drei Prozent Wearables verwendeten. Hier ist jedoch sehr deutlich zwischen den Betriebssystemen zu unterscheiden, die unter anderem auch für die Entwicklung von Apps eine Rolle spielen. So nutzen 81 Prozent Android, wohingegen lediglich 14 Prozent mit dem Apple-Betriebssystem IOS laufen, was ein deutliches Nutzer-Minus zum Vorjahr darstellt. Insgesamt verwendeten 68 Prozent der über 14 Jahre alten, bundesdeutschen Bevölkerung einen mobilen Internetzugang. 82 Prozent dieser Nutzer sind dabei täglich online (ePatient RSD 2016). Eine Tatsache, die vordergründig gerade für präventions- und therapieunterstützende Apps als positiv erscheint. Dabei sind jedoch verschiedene soziale Aspekte genau zu beachten, da eine deutliche geringere Nutzung bei steigendem Alter ebenso zu verzeichnen wie ist bei einem niedrigen Bildungsgrad. Die gleiche Aussage trifft auch auf die digitale Kompetenz zu (ePatient RSD 2016). Beides sind daher Aspekte, die bei der Entwicklung von Gesundheits-Apps keineswegs vernachlässigt werden dürfen. Um hier eine fehlgeleitete Investition beziehungsweise eine nicht-kundenzentrierte Kommunikation zu vermeiden, müssen daher genau die Altersstruktur und die Zusammensetzung nach Bildungsgrad der jeweiligen Indikation berücksichtigt werden, um die richtigen Kanäle zu verwenden. Aus Sicht einer erfolgreichen Kundenorientierung stellt auch das Missverhältnis zwischen der technischen Begeisterung für Wearables auf Herstellerund Gesundheitsanbieterseite und der sehr geringen Akzeptanz in der deutlichen Mehrheit der Bevölkerung ein großes Risiko dar. Es kristallisiert sich daher heraus, dass die Anbieter sich sehr genau mit ihren jeweiligen Zielgruppen und deren Informationsverhalten beschäftigen müssen, um sich für die richtige Vorgehensweise und die damit verbundenen Instrumente zu entscheiden.

\section{Trends und Entwicklung}

Unter dem Aspekt der Kundenorientierung stellt sich bei der Betrachtung des Gesundheitsmarktes auch automatisch die Frage, inwieweit das riesige Angebot an Gesundheitsapps aus Sicht der Patienten ohne Navigationshilfe nicht zu unübersichtlich und ineffektiv ist. Auch ohne dass diese Frage geklärt ist, werden die Nutzerzahlen von Gesundheitsapps weltweit weiter steigen. Die FDA, die bereits 2015 eine Qualifizierung dieser Art von Apps vorgenommen hat, rechnet damit, dass bis 2018 etwa 50 Prozent der bis dann weltweit 3,4 Milliarden Smartphone- und Tabletnutzer solche Tools verwenden werden. Ein Großteil der Gesundheitsapps wird dabei aber im zweiten Gesundheitsmarkt, dem Fitness- und Wellnessbereich, zum Einsatz kommen, in dem bereits heute ein großer Teil der Zielgruppe ihr Smartphone beispielsweise beim Sport verwendet. Medical Apps, die die Beobachtung und Analyse der Gesundheitsparameter des jeweiligen Nutzers beziehungsweise dessen therapiekonformes Verhalten unterstützen, werden davon profitieren, dass auch in der wachsenden Gruppe der über 5o-Jährigen die Verwendung von Smartphones und Tablets immer weiter zunimmt. Entscheidend für den Erfolg der Gesundheitsapps wird in diesem Zusammenhang auch die Berücksichtigung der weiteren Entwicklung der Nutzung 
der verschiedenen Betriebssysteme und die Einbindung der ePatienten bei der Entwicklung von Apps, Kommunikationsinstrumenten und Hilfsmitteln sein. Wenn man eher aus Sicht des technisch machbaren und weniger aus Sicht der Nutzer Hilfsmittel für die Gesundheitskommunikation entwirft, muss man sich zumindest die Frage stellen, inwieweit zurzeit ein Fokussieren auf Waerables als sinnvoll erscheint, wenn diese nicht eine deutliche Erhöhung der Akzeptanz bei den Nutzern erfahren. Eine stets höhere Akzeptanz bei Betroffenen erfahren dagegen weiterhin sogenannte Patient Opinion Leader, die verstärkt Blogs bzw. Webseiten zu ihren Erkrankungen unterhalten. Eine Frage wird jedoch weiterhin offen bleiben, nämlich inwieweit künstliche Intelligenzen den ganzen Markt revolutionieren und damit das Leben nicht nur der ePatienten deutlich verändern werden.

\section{Literatur}

Altendorfer L (2017) Neue Formate der digitalen Gesundheitskommunikation, 1. Auflage, Nomos Baden-Baden Brandt M (2017) Smartphone wird wichtigster Online-Zugang, URL: https://de.statista.com/infografik/9872/ prognose-zur-verteilung-des-weltweiten-ip-traffics/?xing_share=news (abgerufen am 20.06.2017)

Breisach T (2016) Health Economics: Making communications on maternal nutrition work. In: Sight and Life Magazine, lahrgang 30, Nr. 2, S. 55 ff.

ePatient Monitor (2011) Indikationsbezogenes Nutzungsverhalten im (Social) Web 2010/2011, eine quantitative und qualitative Gemeinschaftsstudie zu 15 Indikationen von azionare und Vendus, Düsseldorf

ePatient RSD (2016) Digitaler Gesundheitsmarkt: Basisdaten 2017, Die Planungs- und Entscheidungsgrundlage für digitalen Zielgruppen, Ein Marktforschungsprodukt der EPatient RSD GmbH, Berlin

Presseinfo BITKOM vom 16.12.2010 https://www.pressebox.de/pressemitteilung/bitkom-bundesverband-informationswirtschaft-telekommunikation-und-neue-medien-ev/19-Millionen-Deutsche-suchen-GesundheitsRat-im-Web/boxid/395709 (abgerufen am 10. April 2017)

Schachinger A (2014) Der digitale Patient: Analyse eines neuen Phänomens der partizipativen Vernetzung und Kollaboration von Patienten im Internet, 1. Auflage, Nomos Baden-Baden

Wiesehöfer I (2008) Selbstsüchtige Mütter und gefühllose Väter? Bemerkungen zur Ernährung und zum Tod von Neugeborenen in der Antike. In: Mauritsch P et al, Antike Lebenswelten - Konstanz - Wandel Wirkungsmacht, Wiesbaden, S. 503ff.

www.fda.gov/downloads/MedicalDevices/DeviceRegulationandGuidance/GuidanceDocuments/UCM263366. pdf (abgerufen am 10. April 2017)

www.fda.gov/MedicalDevices/DigitalHealth/MobileMedicalApplications/default.htm (abgerufen am 10. April 2017) 